\title{
INTUITIONISTIC FUZZY PROXIMITY SPACES
}

\author{
SEOK JONG LEE and EUN PYO LEE
}

\author{
Received 1 October 2003
}

\begin{abstract}
We introduce the concept of the intuitionistic fuzzy proximity as a generalization of fuzzy proximity, and investigate its properties. Also we investigate the relationship among intuitionistic fuzzy proximity and fuzzy proximity, and intuitionistic fuzzy topology.
\end{abstract}

2000 Mathematics Subject Classification: 54A40, 54E05.

1. Introduction. As a generalization of fuzzy sets, the concept of intuitionistic fuzzy sets was introduced by Atanassov [1]. Recently, Çoker and his colleagues [2, 3, 4] introduced the concept of intuitionistic fuzzy topology which is a generalization of fuzzy topology.

Katsaras [5, 6] introduced the concept of fuzzy proximity, and studied the relationship between fuzzy topology and fuzzy proximity. Liu [9] introduced the concept of $L$-fuzzy proximity for a lattice $L$, and Liu and Luo [10] studied the relation between $L$-fuzzy proximity and $L$-fuzzy uniformity. Also, Khare [7] studied the relationship between classical and fuzzy proximities.

In [8], we studied the relationship between fuzzy topology and intuitionistic fuzzy topology.

In this paper, we introduce the concept of the intuitionistic fuzzy proximity as a generalization of fuzzy proximity, and investigate its properties. Also we investigate the relationship among intuitionistic fuzzy proximity and fuzzy proximity, and intuitionistic fuzzy topology. Moreover, we find an adjunction between intuitionistic fuzzy proximity spaces and fuzzy proximity spaces.

2. Preliminaries. In this section, we recall some of the definitions and theorems related to fuzzy proximity and intuitionistic fuzzy topology.

Let $X$ be a nonempty set and $I$ the unit interval $[0,1]$. An intuitionistic fuzzy set $A$ is an ordered pair

$$
A=\left(\mu_{A}, \gamma_{A}\right) \text {, }
$$

where the functions $\mu_{A}: X \rightarrow I$ and $\gamma_{A}: X \rightarrow I$ denote the degree of membership and the degree of nonmembership, respectively, and $\mu_{A}+\gamma_{A} \leq 1$. Let $I(X)$ denote the set of all intuitionistic fuzzy sets in $X$.

Obviously every fuzzy set $\mu_{A}$ in $X$ is an intuitionistic fuzzy set of the form $\left(\mu_{A}, 1-\mu_{A}\right)$.

DEFINITION 2.1 [1]. Let $A=\left(\mu_{A}, \gamma_{A}\right)$ and $B=\left(\mu_{B}, \gamma_{B}\right)$ be intuitionistic fuzzy sets in $X$. Then 
(1) $A \subseteq B$ if and only if $\mu_{A} \leq \mu_{B}$ and $\gamma_{A} \geq \gamma_{B}$;

(2) $A=B$ if and only if $A \subseteq B$ and $B \subseteq A$;

(3) $A^{c}=\left(\gamma_{A}, \mu_{A}\right)$;

(4) $A \cap B=\left(\mu_{A} \wedge \mu_{B}, \gamma_{A} \vee \gamma_{B}\right)$;

(5) $A \cup B=\left(\mu_{A} \vee \mu_{B}, \gamma_{A} \wedge \gamma_{B}\right)$;

(6) $0_{\sim}=(\tilde{0}, \tilde{1})$ and $1_{\sim}=(\tilde{1}, \tilde{0})$.

Let $f$ be a map from a set $X$ to a set $Y$. Let $A=\left(\mu_{A}, \gamma_{A}\right)$ be an intuitionistic fuzzy set in $X$ and $B=\left(\mu_{B}, \gamma_{B}\right)$ an intuitionistic fuzzy set in $Y$. Then $f^{-1}(B)$ is an intuitionistic fuzzy set in $X$ defined by

$$
f^{-1}(B)=\left(f^{-1}\left(\mu_{B}\right), f^{-1}\left(\gamma_{B}\right)\right)
$$

and $f(A)$ is an intuitionistic fuzzy set in $Y$ defined by

$$
f(A)=\left(f\left(\mu_{A}\right), 1-f\left(1-\gamma_{A}\right)\right) .
$$

DEFINITION 2.2 [3]. An intuitionistic fuzzy topology on $X$ is a family $\mathcal{T}$ of intuitionistic fuzzy sets in $X$ which satisfies the following properties:

(1) $0 \sim, 1 \sim \in \mathcal{T}$;

(2) if $A_{1}, A_{2} \in \mathscr{T}$, then $A_{1} \cap A_{2} \in \mathcal{T}$;

(3) if $A_{i} \in \mathcal{T}$ for each $i$, then $\bigcup A_{i} \in \mathcal{T}$.

The pair $(X, \mathscr{T})$ is called an intuitionistic fuzzy topological space. Any element of $\mathscr{T}$ is called an intuitionistic fuzzy open set in $X$ and the complement, an intuitionistic fuzzy closed set.

DEFINITION $2.3[2,3]$. Let $(X, \mathscr{T})$ be an intuitionistic fuzzy topological space and $A$ an intuitionistic fuzzy set in $X$. Then the fuzzy closure of $A$ is defined by

$$
\operatorname{cl}(A)=\bigcap\left\{F \mid A \subseteq F, F^{c} \in \mathscr{T}\right\}
$$

and the fuzzy interior of $A$ is defined by

$$
\operatorname{int}(A)=\bigcup\{G \mid A \supseteq G, G \in \mathscr{T}\} .
$$

THEOREM 2.4 [3]. For any intuitionistic fuzzy set $A$ in an intuitionistic fuzzy topological space $(X, \mathcal{T})$, there exist

(1) $\operatorname{int}(A)^{c}=\operatorname{cl}\left(A^{c}\right)$;

(2) $\operatorname{cl}(A)^{c}=\operatorname{int}\left(A^{c}\right)$.

THEOREM 2.5 [2]. Let $(X, \mathcal{T})$ be an intuitionistic fuzzy topological space and $\mathrm{cl}: I(X) \rightarrow$ $I(X)$ the fuzzy closure in $(X, \mathcal{T})$. Then for $A, B \in I(X)$, the following properties hold:

(1) $\operatorname{cl}\left(0_{\sim}\right)=0_{\sim}$;

(2) $A \subseteq \operatorname{cl}(A)$;

(3) $\operatorname{cl}(A \cup B)=\operatorname{cl}(A) \cup \operatorname{cl}(B)$;

(4) $\operatorname{cl}(\operatorname{cl}(A))=\operatorname{cl}(A)$. 
DeFINITION 2.6 [3]. Let $\alpha, \beta \in[0,1]$ and $\alpha+\beta \leq 1$. An intuitionistic fuzzy point $x_{(\alpha, \beta)}$ of $X$ is an intuitionistic fuzzy set in $X$ defined by

$$
x_{(\alpha, \beta)}(y)= \begin{cases}(\alpha, \beta) & \text { if } y=x \\ (0,1) & \text { if } y \neq x\end{cases}
$$

In this case, $x$ is called the support of $x_{(\alpha, \beta)}, \alpha$ the value of $x_{(\alpha, \beta)}$ and $\beta$ the nonvalue of $x_{(\alpha, \beta)}$. An intuitionistic fuzzy point $x_{(\alpha, \beta)}$ is said to belong to an intuitionistic fuzzy set $A=\left(\mu_{A}, \gamma_{A}\right)$ in $X$, denoted by $x_{(\alpha, \beta)} \in A$, if $\alpha \leq \mu_{A}(x)$ and $\beta \geq \gamma_{A}(x)$.

Clearly an intuitionistic fuzzy point can be represented by an ordered pair of fuzzy points as follows:

$$
x_{(\alpha, \beta)}=\left(x_{\alpha}, 1-x_{1-\beta}\right) .
$$

DEFINITION 2.7 [3]. Let $(X, \mathscr{T})$ and $(Y, \mathcal{U})$ be intuitionistic fuzzy topological spaces. Then a map $f: X \rightarrow Y$ is said to be

(1) continuous if $f^{-1}(B)$ is an intuitionistic fuzzy open set in $X$, for each intuitionistic fuzzy open set $B$ in $Y$, or equivalently, $f^{-1}(B)$ is an intuitionistic fuzzy closed set in $X$, for each intuitionistic fuzzy closed set $B$ in $Y$,

(2) open if $f(A)$ is an intuitionistic fuzzy open set in $Y$, for each intuitionistic fuzzy open set $A$ in $X$,

(3) closed if $f(A)$ is an intuitionistic fuzzy closed set in $Y$ for each intuitionistic fuzzy closed set $A$ in $X$,

(4) a homeomorphism if $f$ is bijective, continuous, and open.

Definition 2.8 [5]. A relation $\delta \subseteq I^{X} \times I^{X}$ is called a fuzzy proximity on $X$ if it satisfies the following properties:

(1) $\mu \delta \rho$ implies $\rho \delta \mu$;

(2) $(\mu \vee \rho) \delta \lambda$ if and only if $\mu \delta \lambda$ or $\rho \delta \lambda$;

(3) $\mu \delta \rho$ implies $\mu \neq \tilde{0}$ and $\rho \neq \tilde{0}$;

(4) $\mu \phi \rho$ implies that there exists a $\lambda \subseteq I^{X}$ such that $\mu \phi \lambda$ and $1-\lambda \phi \rho$;

(5) $\mu \wedge \rho \neq \tilde{0}$ implies $\mu \delta \rho$.

3. Intuitionistic fuzzy proximity spaces. We are going to introduce the concept of intuitionistic fuzzy proximity spaces and continuous maps between them.

DEFINITION 3.1. An intuitionistic fuzzy proximity on $X$ is a relation $\delta$ on $I(X)$ satisfying the following properties:

(1) $A \delta B$ implies $B \delta A$;

(2) $(A \cup B) \delta C$ if and only if $A \delta C$ or $B \delta C$;

(3) $A \delta B$ implies $A \neq 0_{\sim}$ and $B \neq 0_{\sim}$;

(4) $A \not B$ implies that there exists an $E \in I(X)$ such that $A \not E$ and $E^{c} \not B$;

(5) $A \cap B \neq 0 \sim$ implies $A \delta B$.

The pair $(X, \delta)$ is called an intuitionistic fuzzy proximity space.

We have easily the following lemma. 
LEMMA 3.2. Let $(X, \delta)$ be an intuitionistic fuzzy proximity space. Then the following properties hold:

(1) if $A \delta B, A_{1} \supseteq A$ and $B_{1} \supseteq B$, then $A_{1} \delta B_{1}$;

(2) $A \delta A$ for each $A \neq 0 \sim$;

(3) $A \delta 1_{\sim}$ if and only if $A \neq 0$.

DEFINITION 3.3. Let $\left(X, \delta_{1}\right)$ and $\left(Y, \delta_{2}\right)$ be two intuitionistic fuzzy proximity spaces and $f: X \rightarrow Y$ a map. Then $f$ is called a continuous map if $A \delta_{1} B$ implies $f(A) \delta_{2} f(B)$.

From the fact that $A \subseteq f^{-1} f(A)$ and $C \supseteq f f^{-1}(C)$, we obtain the following lemma.

LEMMA 3.4. Let $\left(X, \delta_{1}\right)$ and $\left(Y, \delta_{2}\right)$ be two intuitionistic fuzzy proximity spaces and $f: X \rightarrow Y$ a map. Then $f$ is continuous if and only if $C \phi_{2} D$ implies $f^{-1}(C) \phi_{1} f^{-1}(D)$, for each $C, D \in I(Y)$.

THEOREM 3.5. Let $\mathrm{cl}: I(X) \rightarrow I(X)$ be a map satisfying the conditions (1)-(4) of Theorem 2.5. Then there is a unique intuitionistic fuzzy topology $\mathscr{T}$ on $X$ such that $\mathrm{cl}=\mathrm{cl}_{\mathscr{T}}$.

Proof. Let $\mathscr{T}=\left\{A \in I(X) \mid \operatorname{cl}\left(A^{c}\right)=A^{c}\right\}$. First, we will show that $\mathscr{T}$ is an intuitionistic fuzzy topology on $X$.

(i) Clearly, $0_{\sim} \in \mathcal{T}$ and $1_{\sim} \in \mathscr{T}$.

(ii) Let $A, B \in \mathscr{T}$. Then $\operatorname{cl}\left(A^{c}\right)=A^{c}$ and $\operatorname{cl}\left(B^{c}\right)=B^{c}$. So

$$
\operatorname{cl}\left((A \cap B)^{c}\right)=\operatorname{cl}\left(A^{c} \cup B^{c}\right)=\operatorname{cl}\left(A^{c}\right) \cup \operatorname{cl}\left(B^{c}\right)=A^{c} \cup B^{c}=(A \cap B)^{c}
$$

and hence $A \cap B \in \mathscr{T}$.

(iii) Let $A_{\alpha} \in \mathcal{T}$ for all $\alpha \in \Gamma$. Then for each $\alpha \in \Gamma, \operatorname{cl}\left(A_{\alpha}^{c}\right)=A_{\alpha}^{c}$. Note that if $A \subseteq B$ then $\mathrm{cl}(A) \subseteq \operatorname{cl}(B)$. So

$$
\operatorname{cl}\left(\left(\bigcup_{\alpha} A_{\alpha}\right)^{c}\right)=\operatorname{cl}\left(\bigcap_{\alpha} A_{\alpha}^{c}\right) \subseteq \bigcap_{\alpha} \operatorname{cl}\left(A_{\alpha}^{c}\right)=\bigcap_{\alpha} A_{\alpha}^{c}=\left(\bigcup_{\alpha} A_{\alpha}\right)^{c} .
$$

Thus $\operatorname{cl}\left(\left(\bigcup_{\alpha} A_{\alpha}\right)^{c}\right)=\left(\bigcup_{\alpha} A_{\alpha}\right)^{c}$ and hence $\bigcup_{\alpha} A_{\alpha} \in \mathscr{T}$. Therefore, $\mathscr{T}$ is an intuitionistic fuzzy topology on $X$.

Next, we will show that $\mathrm{cl}=\operatorname{cl}_{\mathscr{T}}$. Let $A \in I(X)$. Since $\operatorname{cl}(\operatorname{cl}(A))=\operatorname{cl}(A)$ and $A \subseteq \operatorname{cl}(A)$,

$$
\begin{aligned}
\operatorname{cl}_{\mathscr{T}}(A) & =\bigcap\left\{F \in I(X) \mid A \subseteq F, F^{c} \in \mathscr{T}\right\} \\
& =\bigcap\{F \in I(X) \mid A \subseteq F, \operatorname{cl}(F)=F\} \\
& \subseteq \operatorname{cl}(A) .
\end{aligned}
$$

Consider a set $F$ such that $\operatorname{cl}(F)=F$ and $A \subseteq F$. Then $\operatorname{cl}(A) \subseteq \operatorname{cl}(F)=F$. Thus $\operatorname{cl}(A) \subseteq F$ and hence

$$
\mathrm{cl}(A) \subseteq \bigcap\{F \in I(X) \mid A \subseteq F, \operatorname{cl}(F)=F\}=\operatorname{cl}_{\mathscr{J}}(A) .
$$

Hence $\mathrm{cl}=\mathrm{cl}_{\mathscr{T}}$. 
Finally, we will show that such a $\mathscr{T}$ is unique. Suppose $\mathscr{T}^{*}$ is an intuitionistic fuzzy topology on $X$ such that $\mathrm{cl}=\operatorname{cl}_{\mathscr{T} *}$. Let $A \in \mathscr{T}$. Then $\operatorname{cl}\left(A^{c}\right)=A^{c}$. So $\operatorname{cl}_{\mathscr{T} *}\left(A^{c}\right)=\operatorname{cl}\left(A^{c}\right)=A^{c}$ and hence $A \in \mathscr{T}^{*}$. Also, let $A \in \mathscr{T}^{*}$. Then $\operatorname{cl}\left(A^{c}\right)=\operatorname{cl}_{\mathscr{T}^{*}}\left(A^{c}\right)=A^{c}$ and hence $A \in \mathscr{T}$. Thus $\mathscr{T}=\mathscr{T}^{*}$.

THEOREM 3.6. Let $(X, \delta)$ be an intuitionistic fuzzy proximity space and define a map $\mathrm{cl}: I(X) \rightarrow I(X)$ by

$$
\operatorname{cl}(A)=\bigcap\left\{B^{c} \in I(X) \mid A \not B\right\},
$$

for each $A \in I(X)$. Then the following properties hold:

(1) $A \subseteq \operatorname{cl}(A)$;

(2) $\operatorname{cl}(\operatorname{cl}(A))=\operatorname{cl}(A)$;

(3) $\operatorname{cl}(A \cup B)=\operatorname{cl}(A) \cup \operatorname{cl}(B)$;

(4) $\operatorname{cl}\left(0_{\sim}\right)=0_{\sim}$.

Proof. (1) Let $A=\left(\mu_{A}, \gamma_{A}\right) \in I(X)$. Take any $B=\left(\mu_{B}, \gamma_{B}\right) \in I(X)$ such that $A \varnothing B$. Then $A \cap B=0_{\sim}=(\tilde{0}, \tilde{1})$ and hence $\mu_{A} \wedge \mu_{B}=0$ and $\gamma_{A} \vee \gamma_{B}=1$. So $\mu_{A}+\mu_{B} \leq 1$ and $\gamma_{A}+\gamma_{B} \geq$ 1. Thus $\mu_{B} \leq 1-\gamma_{B} \leq \gamma_{A}$ and $\gamma_{B} \geq 1-\gamma_{A} \geq \mu_{A}$. Hence $B^{c}=\left(\gamma_{B}, \mu_{B}\right) \supseteq\left(\mu_{A}, \gamma_{A}\right)=A$. Therefore,

$$
A \subseteq \bigcap\left\{B^{c} \mid A \phi B\right\}=\operatorname{cl}(A)
$$

(2) It is sufficient to show that $\operatorname{cl}(A) \phi B$ if and only if $A \not B$. If $A \delta B$, then $\operatorname{cl}(A) \delta B$ obviously. Conversely, suppose that $A \Phi B$ and $\operatorname{cl}(A) \delta B$. Then there exists an $E \in I(X)$ such that $B \phi E$ and $E^{c} \not A$. Since $\operatorname{cl}(A) \delta B$ and $E \phi B, \operatorname{cl}(A) \nsubseteq E$ and hence $\mu_{\mathrm{cl}(A)} \ddagger \mu_{E}$ or $\gamma_{\mathrm{cl}(A)} \geq \gamma_{E}$. So there exists an $x \in X$ such that

$$
\mu_{\mathrm{cl}(A)}(x)>\mu_{E}(x) \text { or } \gamma_{\mathrm{cl}(A)}(x)<\gamma_{E}(x) \text {. }
$$

If $\mu_{\mathrm{cl}(A)}(x)>\mu_{E}(x)$, we choose $a \in I$ such that $\mu_{E}(x)<a<\mu_{\mathrm{cl}(A)}(x)$. Define $G: X \rightarrow I \times I$ by

$$
G(y)= \begin{cases}(0, a) & \text { if } y=x \\ (0,1) & \text { if } y \neq x\end{cases}
$$

Then $G \in I(X)$ and $G \subseteq E^{c}$. If $G \phi A$ then $\operatorname{cl}(A) \subseteq G^{c}$ by the definition of closure and hence $\mu_{\mathrm{cl}(A)}(x) \leq \mu_{G^{c}}(x)=\gamma_{G}(x)=a<\mu_{\mathrm{cl}(A)}(x)$. This is a contradiction. Thus $G \delta A$. Since $G \subseteq E^{c}, A \delta E^{c}$. This is a contradiction to the fact that $E^{c} \phi A$. Hence $\mu_{\mathrm{cl}(A)}(x) \leq \mu_{E}(x)$.

Next, if $\gamma_{\mathrm{cl}(A)}(x)<\gamma_{E}(x)$, we can choose $b \in I$ such that $\gamma_{\mathrm{cl}(A)}(x)<b<\gamma_{E}(x)$. Define $H: X \rightarrow I \times I$ by

$$
H(y)= \begin{cases}(b, 1-b) & \text { if } y=x \\ (0,1) & \text { if } y \neq x\end{cases}
$$


Then $H \in I(X)$. Since $\mu_{H}(x)=b<\gamma_{E}(x)=\mu_{E^{c}}(x)$ and $\gamma_{H}(x)=1-b>1-\gamma_{E}(x) \geq$ $\mu_{E}(x)=\gamma_{E^{c}}(x), H \subseteq E^{c}$. If $H \phi A$ then $\operatorname{cl}(A) \subseteq H^{c}$ by the definition of closure and hence $\gamma_{\mathrm{cl}(A)}(x) \geq \gamma_{H^{c}}(x)=\mu_{H}(x)=b>\gamma_{\mathrm{cl}(A)}(x)$. This is a contradiction. Thus we have $H \delta A$. Since $H \subseteq E^{c}, A \delta E^{c}$. This is a contradiction. In any case, we have a contradiction. So $A \phi B$ implies $\operatorname{cl}(A) \phi B$.

(3) It is easy to show that $\operatorname{cl}(A \cup B) \supseteq \operatorname{cl}(A) \cup \operatorname{cl}(B)$. On the other hand, suppose $\left(\mu_{\mathrm{cl}(A \cup B)}, \gamma_{\mathrm{cl}(A \cup B)}\right)=\operatorname{cl}(A \cup B) \nsubseteq \mathrm{cl}(A) \cup \operatorname{cl}(B)=\left(\mu_{\mathrm{cl}(A)} \vee \mu_{\mathrm{cl}(B)}, \gamma_{\mathrm{cl}(A)} \wedge \gamma_{\mathrm{cl}(B)}\right)$. Then there exists an $x \in X$ such that

$$
\mu_{\mathrm{cl}(A \cup B)}(x)>\mu_{\mathrm{cl}(A)}(x) \vee \mu_{\mathrm{cl}(B)}(x) \text { or } \gamma_{\mathrm{cl}(A \cup B)}(x)<\gamma_{\mathrm{cl}(A)}(x) \wedge \gamma_{\mathrm{cl}(B)}(x) \text {. }
$$

CASE 1. Suppose $\mu_{\mathrm{cl}(A \cup B)}(x)>\mu_{\mathrm{cl}(A)}(x) \vee \mu_{\mathrm{cl}(B)}(x)$. We may assume $\mu_{\mathrm{cl}(A)}(x) \geq$ $\mu_{\mathrm{cl}(B)}(x)$. Let $\mu_{\mathrm{cl}(A \cup B)}(x)=a$. Then $\mu_{\mathrm{cl}(A)}(x)<a$ and hence there exists an $\epsilon>0$ such that $\mu_{\mathrm{cl}(A)}(x)<a-\epsilon$. Since $\mu_{\mathrm{cl}(A)}(x)=\bigwedge\left\{\gamma_{C}(x) \mid C \not \phi A\right\}$, there exists a $C \in I(X)$ such that $C \not A$ and $\gamma_{C}(x)<a-\epsilon$. Note that

$$
\gamma_{C}(x) \geq \mu_{\mathrm{cl}(A)}(x) \geq \mu_{\mathrm{cl}(B)}(x)>\mu_{\mathrm{cl}(B)}(x)-\frac{\epsilon}{2},
$$

and hence $\mu_{\mathrm{cl}(B)}(x)<\gamma_{C}(x)+\epsilon / 2$. Since $\mu_{\mathrm{cl}(B)}(x)=\bigwedge\left\{\gamma_{D}(x) \mid D \phi B\right\}$, there exists a $D \in I(X)$ such that $D \phi B$ and $\gamma_{D}(x)<\gamma_{C}(x)+\epsilon / 2$. Since $(C \cap D) \phi A$ and $(C \cap D) \phi B$, we have $(C \cap D) \phi(A \cup B)$. So, from the definition of closure, we have $\operatorname{cl}(A \cup B) \subseteq(C \cap D)^{c}$. Also $\gamma_{C}(x) \vee \gamma_{D}(x)<\gamma_{C}(x)+\epsilon / 2$. Hence

$$
\begin{aligned}
a & =\mu_{\mathrm{cl}(A \cup B)}(x) \leq \mu_{(C \cap D)^{c}}(x)=\gamma_{C \cap D}(x)=\gamma_{C}(x) \vee \gamma_{D}(x) \\
& <\gamma_{C}(x)+\frac{\epsilon}{2}<a-\epsilon+\frac{\epsilon}{2}=a-\frac{\epsilon}{2} .
\end{aligned}
$$

This is a contradiction.

CASE 2. Suppose $\gamma_{\mathrm{cl}(A \cup B)}(x)<\gamma_{\mathrm{cl}(A)}(x) \wedge \gamma_{\mathrm{cl}(B)}(x)$. We may assume $\gamma_{\mathrm{cl}(A)}(x) \leq$ $\gamma_{\mathrm{cl}(B)}(x)$. Let $\gamma_{\mathrm{cl}(A \cup B)}(x)=a$. Then $a<\gamma_{\mathrm{cl}(A)}(x)$ and hence there exists an $\epsilon>0$ such that $a+\epsilon<\gamma_{\mathrm{cl}(A)}(x)$. Since $\gamma_{\mathrm{cl}(A)}(x)=\bigvee\left\{\mu_{C}(x) \mid C \not A\right\}$, there exists an intuitionistic fuzzy set $C \in I(X)$ such that $C \not A$ and $a+\epsilon<\mu_{C}(x)$. Note that,

$$
\mu_{C}(x) \leq \gamma_{\mathrm{cl}(A)}(x) \leq \gamma_{\mathrm{cl}(B)}(x)<\gamma_{\mathrm{cl}(B)}(x)+\frac{\epsilon}{2},
$$

and hence $\mu_{C}(x)-\epsilon / 2<\gamma_{\mathrm{cl}(B)}(x)$. Since $\gamma_{\mathrm{cl}(B)}(x)=\bigvee\left\{\mu_{D}(x) \mid D \phi B\right\}$, there exists a $D \in I(X)$ such that $D \phi B$ and $\mu_{C}(x)-\epsilon / 2<\mu_{D}(x)$. Since $(C \cap D) \phi A$ and $(C \cap D) \phi B$, we have $(C \cap D) \phi(A \cup B)$. So, from the definition of closure, we have $\operatorname{cl}(A \cup B) \subseteq(C \cap D)^{c}$. Also $\mu_{C}(x)-\epsilon / 2<\mu_{C}(x) \wedge \mu_{D}(x)$. Hence

$$
\begin{aligned}
a & =\gamma_{\mathrm{Cl}(A \cup B)}(x) \geq \gamma_{(C \cap D)^{c}}(x)=\mu_{C \cap D}(x)=\mu_{C}(x) \wedge \mu_{D}(x) \\
& >\mu_{C}(x)-\frac{\epsilon}{2}>a+\epsilon-\frac{\epsilon}{2}=a+\frac{\epsilon}{2} .
\end{aligned}
$$

This is a contradiction.

In any case, we have a contradiction. Therefore $\operatorname{cl}(A \cup B)=\operatorname{cl}(A) \cup \operatorname{cl}(B)$.

(4) Since $1 \sim \phi 0_{\sim}, \operatorname{cl}\left(0_{\sim}\right)=\bigcap\left\{B^{c} \mid B \phi 0_{\sim}\right\}=(0,1)=0_{\sim}$. 
THEOREM 3.7. For an intuitionistic fuzzy proximity space $(X, \delta)$, the family

$$
\mathscr{T}(\delta)=\left\{A \in I(X) \mid \mathrm{cl}\left(A^{c}\right)=A^{c}\right\}
$$

is an intuitionistic fuzzy topology on $X$.

Proof. By Theorems 3.5 and 3.6, the proof follows.

DEFINITION 3.8. The topology $\mathscr{T}(\delta)$ defined in Theorem 3.7 is called the intuitionistic fuzzy topology on $X$ induced by the fuzzy proximity $\delta$.

THEOREM 3.9. Let $\left(X, \delta_{1}\right)$ and $\left(Y, \delta_{2}\right)$ be two intuitionistic fuzzy proximity spaces and $f: X \rightarrow Y$ a continuous map. Then $f:\left(X, \mathcal{T}\left(\delta_{1}\right)\right) \rightarrow\left(Y, \mathscr{T}\left(\delta_{2}\right)\right)$ is continuous with respect to the corresponding intuitionistic fuzzy topologies $\mathscr{T}\left(\delta_{1}\right)$ and $\mathscr{T}\left(\delta_{2}\right)$.

Proof. Let $A \in \mathscr{T}\left(\delta_{2}\right)$. Then $\operatorname{cl}\left(A^{c}\right)=A^{c}$. We will show that $\operatorname{cl}\left(f^{-1}(A)^{c}\right)=$ $f^{-1}(A)^{c}$. Clearly $f^{-1}(A)^{c} \subseteq \operatorname{cl}\left(f^{-1}(A)^{c}\right)$. Conversely, let $B \phi_{2} A^{c}$. Since $f$ is continuous, $f^{-1}(B) \phi_{1} f^{-1}\left(A^{c}\right)=f^{-1}(A)^{c}$. Thus

$$
\operatorname{cl}\left(f^{-1}(A)^{c}\right)=\bigcap\left\{K^{c} \mid K \phi_{1} f^{-1}(A)^{c}\right\} \subseteq f^{-1}(B)^{c} .
$$

Hence for any $B \phi_{2} A^{c}, \operatorname{cl}\left(f^{-1}(A)^{c}\right) \subseteq f^{-1}(B)^{c}$. So, we have

$$
\begin{aligned}
\operatorname{cl}\left(f^{-1}(A)^{c}\right) & \subseteq \bigcap\left\{f^{-1}(B)^{c} \mid B \phi_{2} A^{c}\right\} \\
& =\bigcap\left\{f^{-1}\left(B^{c}\right) \mid B \phi_{2} A^{c}\right\} \\
& =f^{-1}\left(\bigcap\left\{B^{c} \mid B \phi_{2} A^{c}\right\}\right) \\
& =f^{-1}\left(\operatorname{cl}\left(A^{c}\right)\right)=f^{-1}\left(A^{c}\right)=f^{-1}(A)^{c} .
\end{aligned}
$$

Thus $\operatorname{cl}\left(f^{-1}(A)^{c}\right)=f^{-1}(A)^{c}$. Hence $f^{-1}(A)$ is open. Therefore, $f$ is a continuous map.

4. The $\delta$-neighborhood in the intuitionistic fuzzy proximity. In this section, we will introduce the notion of the $\delta$-neighborhood in the intuitionistic fuzzy proximity.

DeFinition 4.1. Let $(X, \delta)$ be an intuitionistic fuzzy proximity space. For $A, B \in$ $I(X)$, the intuitionistic fuzzy set $B$ is said to be a $\delta$-neighborhood of $A$ (in symbols $A \ll B)$ if $A \phi B^{c}$.

Clearly, we know that if $A \ll B$, then $A \subseteq B$.

THEOREM 4.2. Let $(X, \delta)$ be an intuitionistic fuzzy proximity space and $A, B \in I(X)$. Then the following properties hold:

(1) $A \ll B$ if and only if $\operatorname{cl}(A) \ll B$;

(2) if $A \ll B$, then there exists an element $G$ of the intuitionistic fuzzy topology $\mathscr{T}(\delta)$ induced by $\delta$ on $X$ such that $A \subseteq G \subseteq B$;

(3) if $A \phi B$, then there are $E, F \in I(X)$ such that $A \ll E, B \ll F$, and $E \phi F$. 
Proof. (1) It follows from the fact that $A \not B$ if and only if $\operatorname{cl}(A) \phi B$ (see the proof of Theorem 3.6(2)).

(2) Let $A \ll B$. Then $A \not B^{c}$ and hence

$$
\operatorname{cl}\left(B^{c}\right)=\bigcap\left\{K^{c} \mid K \not B^{c}\right\} \subseteq A^{c} .
$$

Thus $B^{c} \subseteq \operatorname{cl}\left(B^{c}\right) \subseteq A^{c}$. Put $G=\operatorname{cl}\left(B^{c}\right)^{c}$. Note that

$$
\operatorname{cl}\left(G^{c}\right)=\operatorname{cl}\left(\operatorname{cl}\left(B^{c}\right)\right)=\operatorname{cl}\left(B^{c}\right)=G^{c} .
$$

Hence $G \in \mathscr{T}(\delta)$ and $A \subseteq G \subseteq B$.

(3) Suppose $A \not B$. Then there exists an $E \in I(X)$ such that $A \not E^{c}$ and $E \phi B$. Since $B \phi E$, there exists an $F \in I(X)$ such that $B \phi F^{c}$ and $F \phi E$. Thus there are $E, F \in I(X)$ such that $A \ll E, B \ll F$, and $E \phi F$.

THEOREM 4.3. Let $(X, \delta)$ be an intuitionistic fuzzy proximity space. Then the relation $\ll$ on $I(X)$ has the following properties:

(1) $1_{\sim} \ll 1_{\sim}$;

(2) $A \ll B$ implies $A \cap B^{c}=0_{\sim}$;

(3) if $A_{1} \subseteq A \ll B \subseteq B_{1}$, then $A_{1} \ll B_{1}$;

(4) $A \ll B_{1} \cap B_{2}$ if and only if $A \ll B_{1}$ and $A \ll B_{2}$;

(5) $A \ll B$ implies $B^{c} \ll A^{c}$;

(6) if $A \ll B$, then there exists a set $E \in I(X)$ such that $A \ll E \ll B$.

Proof. (1) Since $1_{\sim} \phi 0_{\sim}=1_{\sim}^{c}$, we have $1_{\sim} \ll 1_{\sim}$.

(2) Let $A \ll B$. Then $A \phi B^{c}$ and hence $A \cap B^{c}=0 \sim$.

(3) Let $A_{1} \subseteq A \ll B \subseteq B_{1}$. Then $A \not B^{c}$. Since $A_{1} \subseteq A$ and $B_{1}^{c} \subseteq B^{c}$, we have $A_{1} \phi B_{1}^{c}$ and hence $A_{1} \ll B_{1}$.

(4)

$$
\begin{aligned}
A \ll B_{1} \cap B_{2} & \Longleftrightarrow A \phi\left(B_{1} \cap B_{2}\right)^{c}=B_{1}^{c} \cup B_{2}^{c} \\
& \Longleftrightarrow A \phi B_{1}^{c} \text { and } A \phi B_{2}^{c} \\
& \Longleftrightarrow A \ll B_{1} \text { and } A \ll B_{2} .
\end{aligned}
$$

(5) Let $A \ll B$. Then $A \phi B^{c}$ and hence $B^{c} \phi A=\left(A^{c}\right)^{c}$. Thus $B^{c} \ll A^{c}$.

(6) Let $A \ll B$. Then $A \not B^{c}$. Thus there exists a set $E \in I(X)$ such that $A \not E^{c}$ and $E \phi B^{c}$ and hence $A \ll E \ll B$.

THEOREM 4.4. Let $\ll$ be a relation on $I(X)$ satisfying (1)-(6) of the above theorem. Then the relation $\delta$ on $I(X)$, defined by $A \phi B$ if and only if $A \ll B^{c}$, is an intuitionistic fuzzy proximity on $X$. Also, with respect to this intuitionistic fuzzy proximity, $B$ is a $\delta$ neighborhood of $A$ if and only if $A \ll B$. 
Proof. First, we will show that $\delta$ is an intuitionistic fuzzy proximity on $X$.

(1) Let $A \not B$. Then $A \ll B^{c}$ and hence $B \ll A^{c}$. So $B \phi A$.

(2)

$$
\begin{aligned}
A \phi\left(B_{1} \cup B_{2}\right) & \Longleftrightarrow A \ll\left(B_{1} \cup B_{2}\right)^{c}=B_{1}^{c} \cap B_{2}^{c} \\
& \Leftrightarrow A \ll B_{1}^{c} \text { and } A \ll B_{2}^{c} \\
& \Longleftrightarrow A \phi B_{1} \text { and } A \phi B_{2} .
\end{aligned}
$$

(3) Let $B=0_{\sim}$. Since $A \subseteq 1_{\sim} \ll 1_{\sim} \subseteq 1_{\sim}$, we have $A \ll 1_{\sim}=\left(0_{\sim}\right)^{c}=B^{c}$ and hence $A \varnothing B$.

(4) Let $A \not B$. Then $A \ll B^{c}$. Thus there exists an $E \in I(X)$ such that $A \ll E \ll B^{c}$. So $A \ll\left(E^{c}\right)^{c}$ and $E \ll B^{c}$. Hence $A \phi E^{c}$ and $E \phi B$.

(5) If $A \phi B$, then $A \ll B^{c}$ and hence $A \cap B=A \cap\left(B^{c}\right)^{c}=0_{\sim}$.

Therefore $\delta$ is an intuitionistic fuzzy proximity on $X$. Clearly, $B$ is a $\delta$-neighborhood of $A$ if and only if $A \phi B^{c}$ if and only if $A \ll\left(B^{c}\right)^{c}=B$.

THEOREM 4.5. Let $(X, \delta)$ be an intuitionistic fuzzy proximity space and $A \in I(X)$. Then

$$
\operatorname{cl}(A)=\bigcap\{B \mid A \ll B\} .
$$

Proof. Let $K=\bigcap\{B \mid A \ll B\}$. Take any $B$ with $A \ll B$. Then $\operatorname{cl}(A) \ll B$ and hence $\operatorname{cl}(A) \subseteq B$. Thus

$$
\operatorname{cl}(A) \subseteq \bigcap\{B \mid A \ll B\}=K .
$$

Conversely, suppose $\operatorname{cl}(A) \nsupseteq K$. Then $\mu_{\mathrm{cl}(A)} ¥ \mu_{K}$ or $\gamma_{\mathrm{cl}(A)} \npreceq \gamma_{K}$ and hence there exists an $x \in X$ such that

$$
\mu_{\mathrm{cl}(A)}(x)<\mu_{K}(x) \text { or } \gamma_{\mathrm{cl}(A)}(x)>\gamma_{K}(x) \text {. }
$$

Suppose $\mu_{\mathrm{cl}(A)}(x)<\mu_{K}(x)$. Let $\mu_{K}(x)=a$. Then there exists an $\epsilon>0$ such that $\mu_{\mathrm{cl}(A)}(x)$ $<a-\epsilon$. Since $\mu_{\mathrm{cl}(A)}(x)=\bigwedge\left\{\gamma_{B}(x) \mid A \phi B\right\}$, there exists an $E \in I(X)$ such that $A \Phi E$ and $\gamma_{E}(x)<a-\epsilon$. Since $A \not\left(E^{c}\right)^{c}, A \ll E^{c}$ and hence $K=\bigcap\{B \mid A \ll B\} \subseteq E^{c}$. Thus

$$
a=\mu_{K}(x) \leq \mu_{E^{c}}(x)=\gamma_{E}(x)<a-\epsilon .
$$

This is a contradiction. Next, suppose $\gamma_{\mathrm{cl}(A)}(x)>\gamma_{K}(x)$. Let $\gamma_{K}(x)=a$. Then there exists an $\epsilon>0$ such that $\gamma_{\mathrm{cl}(A)}(x)>a+\epsilon$. Since $\gamma_{\mathrm{cl}(A)}(x)=\bigvee\left\{\mu_{B}(x) \mid A \not B\right\}$, there exists an $F \in I(X)$ such that $A \not F$ and $\mu_{F}(x)>a+\epsilon$. Since $A \phi\left(F^{c}\right)^{c}, A \ll F^{c}$ and hence $K=\bigcap\{B \mid A \ll B\} \subseteq F^{c}$. Thus

$$
a=\gamma_{K}(x) \geq \gamma_{F c}(x)=\mu_{F}(x)>a+\epsilon .
$$

This is a contradiction. 
5. Category of intuitionistic fuzzy proximity spaces. We knew the relationship between fuzzy topological spaces and fuzzy proximity spaces (see [5, 6]). The relationship between fuzzy topological spaces and intuitionistic fuzzy topological spaces had been studied in [8]. Also we have had the relationship between intuitionistic fuzzy proximity spaces and intuitionistic fuzzy topological spaces in Theorems 3.7 and 3.9. Now, we are going to find a categorical relationship between fuzzy proximity spaces and intuitionistic fuzzy proximity spaces.

Let FProx be the category of all fuzzy proximity spaces and proximity maps and IFProx the category of all intuitionistic fuzzy proximity spaces and continuous maps.

THEOREM 5.1. Define $F:$ FProx $\rightarrow$ IFProx $b y$

$$
F(X, \sigma)=(X, \delta), \quad F(f)=f
$$

where for $A, B \in I(X), A \delta B$ if and only if $\left(1-\gamma_{A}\right) \sigma\left(1-\gamma_{B}\right)$. Then $F$ is a functor.

Proof. First, we will show that $\delta$ is an intuitionistic fuzzy proximity on $X$.

(1) Clearly, $A \delta B$ implies $B \delta A$.

(2) Note that $1-\gamma_{A \cup B}=1-\left(\gamma_{A} \wedge \gamma_{B}\right)=\left(1-\gamma_{A}\right) \vee\left(1-\gamma_{B}\right)$. Thus

$$
\begin{aligned}
C \delta(A \cup B) & \Longleftrightarrow\left(1-\gamma_{C}\right) \sigma\left(1-\gamma_{A \cup B}\right) \\
& \Longleftrightarrow\left(1-\gamma_{C}\right) \sigma\left[\left(1-\gamma_{A}\right) \vee\left(1-\gamma_{B}\right)\right] \\
& \Longleftrightarrow\left(1-\gamma_{C}\right) \sigma\left(1-\gamma_{A}\right) \text { or }\left(1-\gamma_{C}\right) \sigma\left(1-\gamma_{B}\right) \\
& \Longleftrightarrow C \delta A \text { or } C \delta B .
\end{aligned}
$$

(3) Since $\left(1-\gamma_{A}\right) \phi \tilde{0}=\left(1-\gamma_{0 \sim}\right)$, we have $A \not 0_{\sim}$.

(4) Let $A \not B$. Then $\left(1-\gamma_{A}\right) \phi\left(1-\gamma_{B}\right)$. Then there exists a $\rho \in I^{X}$ such that $\left(1-\gamma_{A}\right) \phi \rho$ and $(1-\rho) \phi\left(1-\gamma_{B}\right)$. Let $E=\left(\mu_{E}, \gamma_{E}\right)=(\rho, 1-\rho)$. Then $E \in I(X)$. Since $\left(1-\gamma_{A}\right) \phi \rho=$ $\left(1-\gamma_{E}\right), A \phi E$. Note that $1-\gamma_{E^{c}}=1-\mu_{E}=1-\rho$ and $(1-\rho) \phi\left(1-\gamma_{B}\right)$. So $E^{c} \not B$.

(5) Let $A \cap B \neq 0_{\sim}$. Suppose $\gamma_{A} \vee \gamma_{B}=\tilde{1}$. Then

$$
\tilde{1}=\gamma_{A} \vee \gamma_{B} \leq\left(1-\mu_{A}\right) \vee\left(1-\mu_{B}\right)=1-\left(\mu_{A} \wedge \mu_{B}\right)
$$

and hence $\mu_{A} \wedge \mu_{B}=\tilde{0}$. So $A \cap B=0_{\sim}$. This is a contradiction. Thus $\gamma_{A} \vee \gamma_{B} \neq 1$. Hence $\left(1-\gamma_{A}\right) \wedge\left(1-\gamma_{B}\right)=1-\left(\gamma_{A} \vee \gamma_{B}\right) \neq 0$. Thus $\left(1-\gamma_{A}\right) \sigma\left(1-\gamma_{B}\right)$ and hence $A \delta B$. Therefore $(X, \delta)$ is an intuitionistic fuzzy proximity space.

Next, we will show that if $f:\left(X, \sigma_{1}\right) \rightarrow\left(Y, \sigma_{2}\right)$ is a proximity map, then $f:\left(X, \delta_{1}\right) \rightarrow$ $\left(Y, \delta_{2}\right)$ is a continuous map. Let $C, D \in I(Y)$ and $C \phi_{2} D$. Then $\left(1-\gamma_{C}\right) \phi_{2}\left(1-\gamma_{D}\right)$. Since $f:\left(X, \sigma_{1}\right) \rightarrow\left(Y, \sigma_{2}\right)$ is a proximity map, $f^{-1}\left(1-\gamma_{C}\right) \phi_{1} f^{-1}\left(1-\gamma_{D}\right)$. Note that

$$
\begin{aligned}
& f^{-1}\left(1-\gamma_{C}\right)=1-f^{-1}\left(\gamma_{C}\right)=1-\gamma_{f^{-1}(C)}, \\
& f^{-1}\left(1-\gamma_{D}\right)=1-f^{-1}\left(\gamma_{D}\right)=1-\gamma_{f^{-1}(D)} .
\end{aligned}
$$

So $\left[1-\gamma_{f^{-1}(C)}\right] \phi_{1}\left[1-\gamma_{f^{-1}(D)}\right]$ and hence $f^{-1}(C) \phi_{1} f^{-1}(D)$. Therefore $f:\left(X, \delta_{1}\right) \rightarrow$ $\left(Y, \delta_{2}\right)$ is a continuous map. In all, $F$ is a functor. 
THEOREM 5.2. Define $G:$ IFProx $\rightarrow$ FProx $b y$

$$
G(X, \delta)=(X, \sigma), \quad G(f)=f,
$$

where for $v, \rho \in I^{X}, v \sigma \rho$ if and only if $(0,1-v) \delta(0,1-\rho)$. Then $G$ is a functor.

Proof. First, we will show that $\sigma$ is a fuzzy proximity on $X$.

(1) Clearly, if $v \sigma \rho$, then $\rho \sigma v$.

(2) Note that $(0,1-(v \vee \rho))=(0,(1-v) \wedge(1-\rho))=(0,1-v) \cup(0,1-\rho)$. Thus

$$
\begin{aligned}
\lambda \sigma(\nu \vee \rho) & \Longleftrightarrow(0,1-\lambda) \delta(0,1-(\nu \vee \rho)) \\
& \Longleftrightarrow(0,1-\lambda) \delta[(0,1-v) \cup(0,1-\rho)] \\
& \Longleftrightarrow(0,1-\lambda) \delta(0,1-v) \text { or }(0,1-\lambda) \delta(0,1-\rho) \\
& \Longleftrightarrow \lambda \sigma v \text { or } \lambda \sigma \rho .
\end{aligned}
$$

(3) Since $(0,1-v) \phi 0_{\sim}=(0,1-0)$, we have $v \phi \tilde{0}$.

(4) Let $v \phi \rho$. Then $(0,1-v) \phi(0,1-\rho)$. So there exists a set $E=\left(\mu_{E}, \gamma_{E}\right) \in I(X)$ such that

$$
(0,1-v) \phi\left(\mu_{E}, \gamma_{E}\right), \quad\left(\gamma_{E}, \mu_{E}\right) \phi(0,1-\rho) .
$$

Since $\left(0,1-\mu_{E}\right) \subseteq\left(\mu_{E}, \gamma_{E}\right)$ and $\left(0, \mu_{E}\right) \subseteq\left(\gamma_{E}, \mu_{E}\right)$,

$$
(0,1-v) \phi\left(0,1-\mu_{E}\right), \quad\left(0, \mu_{E}\right) \phi(0,1-\rho) .
$$

Thus there exists a set $\mu_{E} \in I^{X}$ such that $\nu \phi \mu_{E}$ and $1-\mu_{E} \phi \rho$.

(5) Let $v \wedge \rho \neq \tilde{0}$. Then

$$
(0,1-v) \cap(0,1-\rho)=(0,1-(\nu \wedge \rho)) \neq 0 \sim
$$

So $(0,1-v) \delta(0,1-\rho)$ and hence $v \sigma \rho$. Therefore $(X, \sigma)$ is a fuzzy proximity space.

Next, we will show that if $f:\left(X, \delta_{1}\right) \rightarrow\left(Y, \delta_{2}\right)$ is a continuous map then $f:\left(X, \sigma_{1}\right) \rightarrow$ $\left(Y, \sigma_{2}\right)$ is a proximity map. Let $\nu, \rho \in I^{Y}$ and $\nu \phi_{2} \rho$. Then $(0,1-v) \phi_{2}(0,1-\rho)$. Since $f:\left(X, \delta_{1}\right) \rightarrow\left(Y, \delta_{2}\right)$ is a continuous map, $f^{-1}((0,1-v)) \phi_{1} f^{-1}((0,1-\rho))$. Note that

$$
\begin{aligned}
& f^{-1}((0,1-v))=\left(f^{-1}(0), f^{-1}(1-v)\right)=\left(0,1-f^{-1}(v)\right), \\
& f^{-1}((0,1-\rho))=\left(f^{-1}(0), f^{-1}(1-\rho)\right)=\left(0,1-f^{-1}(\rho)\right) .
\end{aligned}
$$

So $\left(0,1-f^{-1}(\nu)\right) \phi_{1}\left(0,1-f^{-1}(\rho)\right)$ and hence $f^{-1}(\nu) \phi_{1} f^{-1}(\rho)$. Thus $f:\left(X, \sigma_{1}\right) \rightarrow$ $\left(Y, \sigma_{2}\right)$ is a proximity map. In all, $G$ is a functor.

THEOREM 5.3. The functor $F:$ FProx $\rightarrow$ IFProx is a left adjoint of the functor $G:$ IFProx $\rightarrow$ FProx.

Proof. First, we will show that for any $(X, \sigma) \in$ FProx, $1_{X}:(X, \sigma) \rightarrow G(F(X, \sigma))$ is a proximity map. Let $\nu, \rho \in I^{X}$ and $\nu \sigma \rho$. Then $(0,1-\nu) F(\sigma)(0,1-\rho)$ and hence $\nu G(F(\sigma)) \rho$. 
Next, consider $(Y, \delta) \in$ IFProx and a proximity map $f:(X, \sigma) \rightarrow G(Y, \delta)$. In order to show that $f: F(X, \sigma) \rightarrow(Y, \delta)$ is a continuous map, let $A, B \subseteq I(Y)$ and $A \not B$. Since $\left(0, \gamma_{A}\right) \subseteq\left(\mu_{A}, \gamma_{A}\right)=A$ and $\left(0, \gamma_{B}\right) \subseteq\left(\mu_{B}, \gamma_{B}\right)=B,\left(0, \gamma_{A}\right) \phi\left(0, \gamma_{B}\right)$ and hence $(1-$ $\left.\gamma_{A}\right) G(\phi)\left(1-\gamma_{B}\right)$. Since $f:(X, \sigma) \rightarrow G(Y, \delta)$ is a proximity map, $f^{-1}\left(1-\gamma_{A}\right) \phi f^{-1}\left(1-\gamma_{B}\right)$. Note that

$$
\begin{aligned}
f^{-1}\left(1-\gamma_{A}\right) & =1-f^{-1}\left(\gamma_{A}\right)=1-\gamma_{f^{-1}(A)}, \\
f^{-1}\left(1-\gamma_{B}\right) & =1-f^{-1}\left(\gamma_{B}\right)=1-\gamma_{f^{-1}(B)} .
\end{aligned}
$$

So $\left[1-\gamma_{f^{-1}(A)}\right] \phi\left[1-\gamma_{f^{-1}(B)}\right]$ and hence $f^{-1}(A) F(\phi) f^{-1}(B)$. Thus $f: F(X, \sigma) \rightarrow(Y, \delta)$ is a continuous map. Therefore $1_{X}$ is a $G$-universal map for $(X, \sigma)$ in FProx.

ACKNOWLEDGMENT. This work was supported by Grant no. R01-2002-000-00315-0 from the Basic Research Program of the Korea Science \& Engineering Foundation.

\section{REFERENCES}

[1] K. T. Atanassov, Intuitionistic fuzzy sets, Fuzzy Sets and Systems 20 (1986), no. 1, 87-96.

[2] D. Çoker, An introduction to intuitionistic fuzzy topological spaces, Fuzzy Sets and Systems 88 (1997), no. 1, 81-89.

[3] D. Çoker and A. H. Eş, On fuzzy compactness in intuitionistic fuzzy topological spaces, J. Fuzzy Math. 3 (1995), no. 4, 899-909.

[4] H. Gürçay, D. Çoker, and A. H. Eş, On fuzzy continuity in intuitionistic fuzzy topological spaces, J. Fuzzy Math. 5 (1997), no. 2, 365-378.

[5] A. K. Katsaras, Fuzzy proximity spaces, J. Math. Anal. Appl. 68 (1979), no. 1, 100-110.

[6] _ On fuzzy proximity spaces, J. Math. Anal. Appl. 75 (1980), no. 2, 571-583.

[7] M. Khare, A relationship between classical and fuzzy proximities, Fuzzy Sets and Systems 90 (1997), no. 1, 55-59.

[8] S. J. Lee and E. P. Lee, The category of intuitionistic fuzzy topological spaces, Bull. Korean Math. Soc. 37 (2000), no. 1, 63-76.

[9] W. J. Liu, Fuzzy proximity spaces redefined, Fuzzy Sets and Systems 15 (1985), no. 3, 241248.

[10] Y.-M. Liu and M.-K. Luo, Fuzzy Topology, Advances in Fuzzy Systems-Applications and Theory, vol. 9, World Scientific Publishing, New Jersey, 1997.

Seok Jong Lee: Department of Mathematics, Chungbuk National University, Cheongju 361-763, Korea

E-mail address: sj1@cbnu.ac.kr

Eun Pyo Lee: Department of Mathematics, Seonam University, Namwon 590-711, Korea

E-mail address: eplee@seonam. ac.kr 


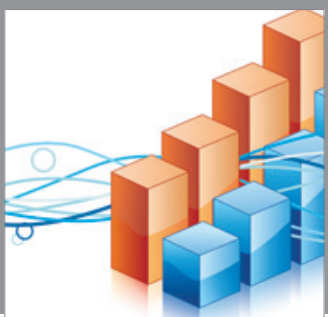

Advances in

Operations Research

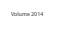

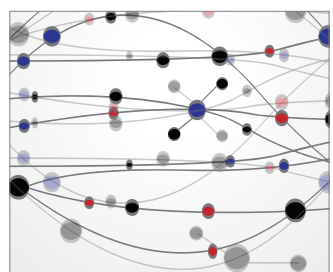

\section{The Scientific} World Journal
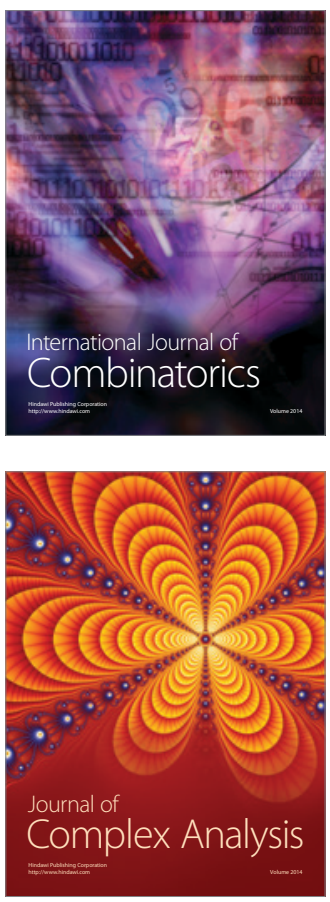

International Journal of

Mathematics and

Mathematical

Sciences
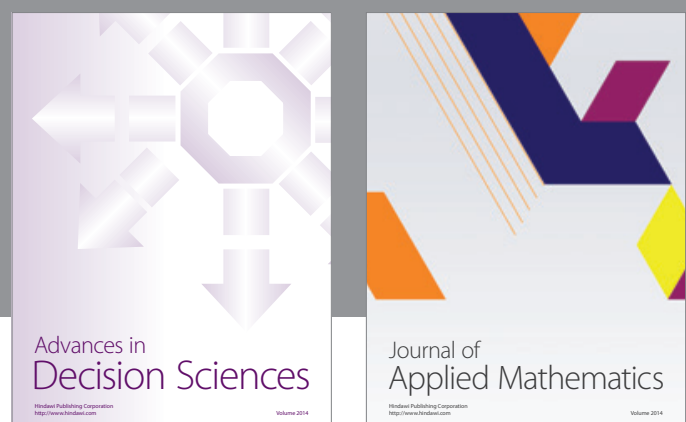

Journal of

Applied Mathematics
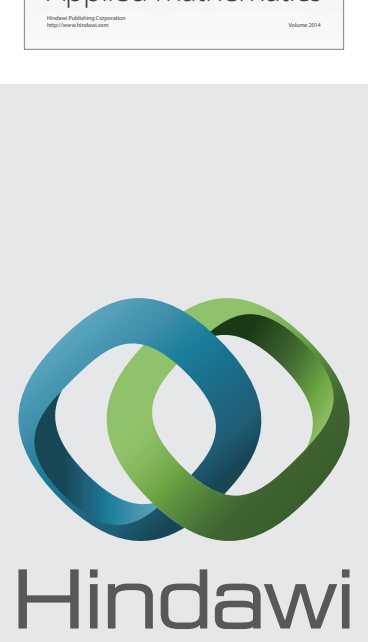

Submit your manuscripts at http://www.hindawi.com
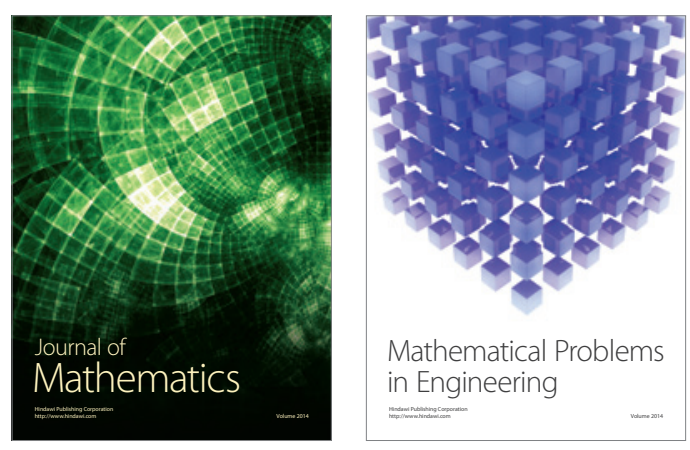

Mathematical Problems in Engineering
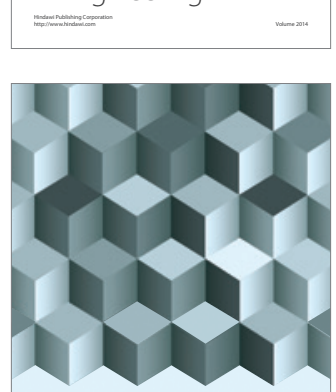

Journal of

Function Spaces
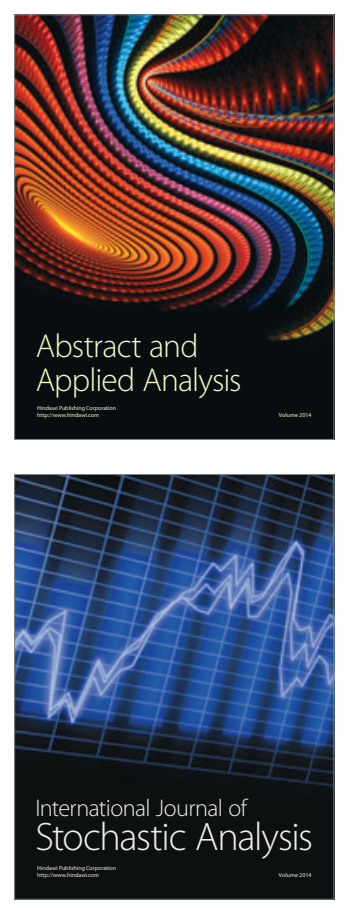

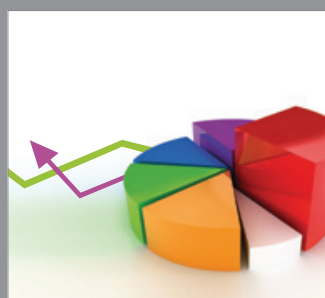

ournal of

Probability and Statistics

Promensencen
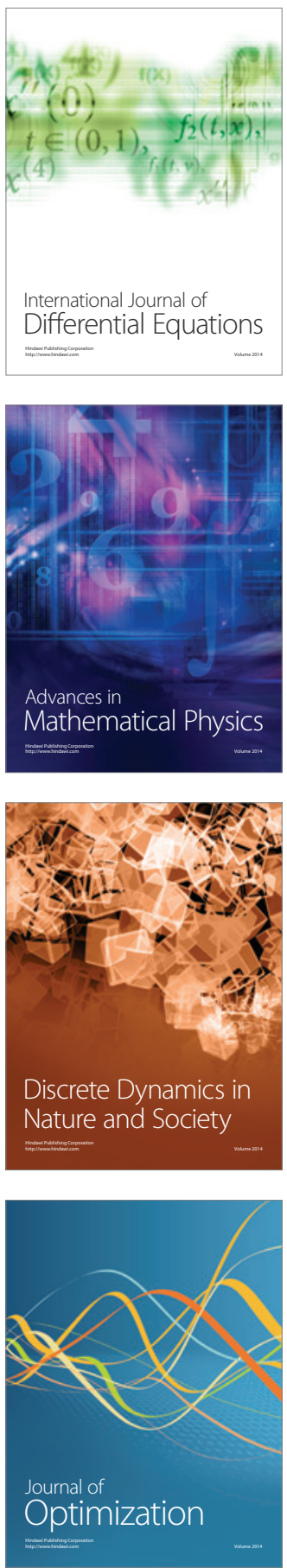